\title{
DESIGNING VEHICLES, INSIDE OUT
}

Automotive design is majorly influenced by aggressive customer demands and stringent regulatory changes in fuel efficiency, safety and sustainability. Albeit fuel efficiency and safety can be managed by innovating design of specific sub-systems, sustainability encompasses all aspects of cradle-to-grave in a vehicle - including green manufacturing, supply chain, logistics and other activities in new and current product launch.

Today, design and styling of vehicles is more welcoming towards lowering coefficient of drag and improving pedestrian safety. The coefficient of drag directly affects fuel efficiency of a vehicle. Lower the value, better is the ability in overcoming resistive forces while driving, implying lesser drag forces and resulting in higher traction power to propel the vehicle with lesser fuel usage. Besides, a lowered hood design, creases and a correlated bumper styling provides higher pedestrian safety in case of an impact. Reducing pedestrian impact risk enhances the brand value in vehicle safety ratings. On the other hand, lowering this coefficient through a stylish design creates manufacturing challenges and negatively influences the cost of body-in-white for mass manufacturing. This cost affect can seriously affect sustainability of mass market vehicle programmes.

The above cause-affect cycle and its prudence to be optimised have to be strategised by integrating upstream actions that affect downstream outcomes. OEMs have been proactively strategizing on pulling in automotive design as an integrated resourceful exercise of product development rather than a standalone styling activity. Options out of an automotive design exercise are evaluated, risks pre-empted in Life Cycle Assessment and then integrated in design and manufacturing. Many of the upstream and downstream developments are streamlined for sustainable vehicle development.

Unlike the past, virtual automotive design has gained tremendous traction in growth markets as well. This has further helped frugality in product develop- ment since almost all shortlisted styled options can actually be projected in a 1:1 scale for viewing various features, critiquing iterations, and evaluating upstream and downstream correlated outcomes. The need to actually build multiple physical prototype models for visualisation of the vehicle is not necessary and now done only for the final or pre-final option. As such, the multi-axis CNC machines to cut out models do not need to be owned by OEMs and can be outsourced along with the appropriate skill sets. This saves considerable time, cost and resources, and reduces product development and manufacturing costs.

\section{HONING SKILLS}

The skill set for automotive design has improved over the years. Designers, in their foundation years, undertake formal training through various programmes in automotive design to build on a creative scientific approach and develop the knack of capturing customer insights. These training programmes are designed to be customer centric and autonomous for understanding advanced development.

Within OEMs, automotive designers are the ones that conceptualise the vehi-

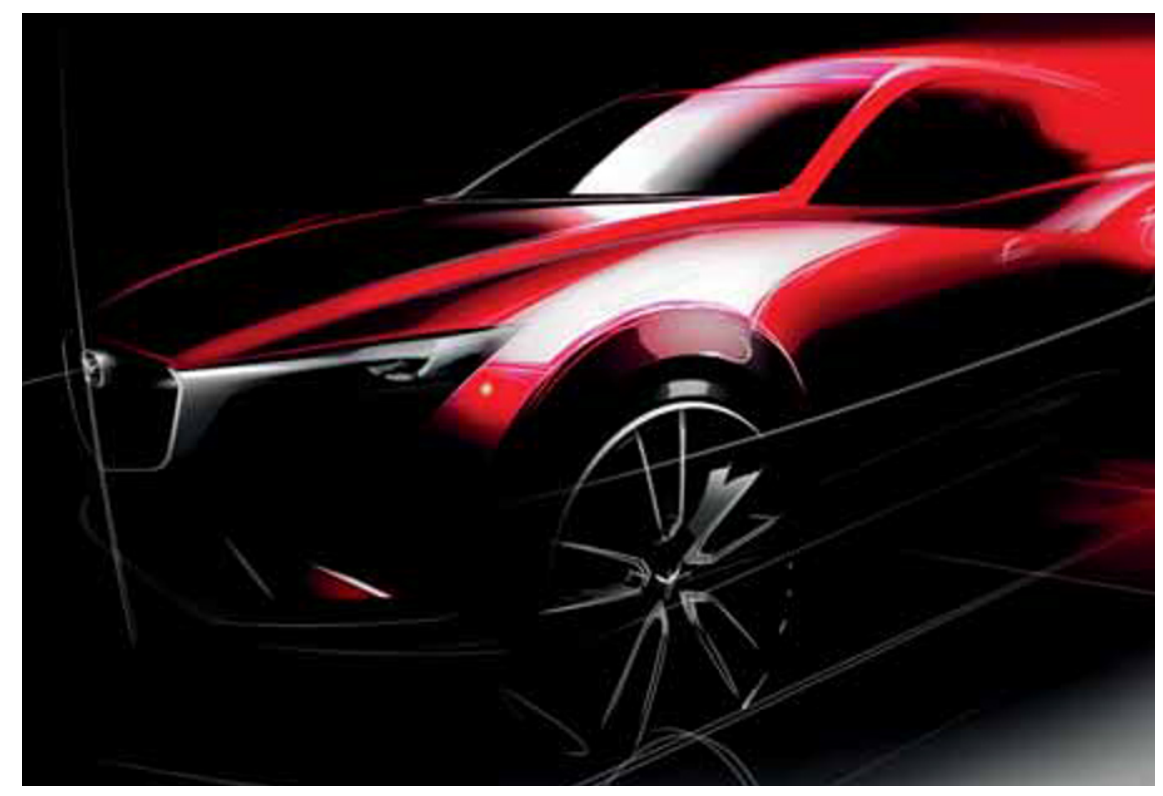

cle and see it shaping through body-inwhite development as they roll out of the production lines through the developmental cycle. In addition, the automotive designers are encouraged to interact with other design and development mobility practitioners within OEM organisations so that they are exposed to a better understanding of vehicle development and engineering. This is useful in designing the vehicle inside out rather than outside in.

Additional incentive to automotive designers besides the above organisational, vehicle and customer aspects is their participation in integrated intellectual property and patent activities. They have the opportunity in becoming coinventors in patents and invention disclosures in vehicle programmes. Automotive design has taken the front seat in product development cycles with the advancement of tools, processes and trained skill sets that have been strategically integrated for sustainable mobility solutions. 